\title{
Nursing Home Quality as a Common Good
}

\author{
David C. Grabowski ${ }^{*}$, \\ Harvard University \\ Jonathan Gruber, and \\ Massachusetts Institute of Technology \\ Joseph J. Angelelli \\ Paraprofessional Healthcare Institute
}

\begin{abstract}
A long-standing assumption among economists is that nursing home quality is common across Medicaid and private-pay patients within a shared facility. However, there has been only limited empirical work addressing this issue. Using a unique individual level panel of residents of nursing homes from seven states, we exploit both within-facility and within-person variation in payer source and quality to examine this issue. We also test the robustness of these results across states with different Medicaid and private-pay rate differentials. Across various identification strategies, our results are consistent with the assumption of common quality across Medicaid and private-paying patients within facilities.
\end{abstract}

\section{Keywords}

Medicaid; quality; nursing home; regulation

\begin{abstract}
A principal goal of the Medicare-Medicaid legislation passed by Congress in 1965 was the incorporation of the elderly and poor into "mainstream" medical care. Despite this objective, poor Medicaid nursing home quality has been an important policy issue for over three decades (U.S. Senate, 1974). A series of studies and reports have argued that Medicaid recipients are generally admitted to lower resource facilities (Institute of Medicine, 2001), and conditional on entry, Medicaid recipients receive worse quality care relative to private-paying patients (U.S. General Accounting Office, 1990). In assessing the impact of payer source on nursing home treatment quality, researchers have basically assumed that poor quality for Medicaid recipients is a cross-facility phenomenon. The research community has generally ignored the idea that there may be within-facility variation by assuming that nursing home quality is a common good enjoyed by all residents within a facility, regardless of payer type (Norton, 2000).
\end{abstract}

There are legal, behavioral and economic arguments underlying the commonality assumption. From a legal perspective, nursing homes certified to accept Medicaid or Medicare residents are required by the Centers for Medicare \& Medicaid Services (CMS) to provide equal quality to all residents, regardless of payer type. The nursing home State Operations Manual published by CMS (formerly the Health Care Financing Administration) states that "facilities must not distinguish between residents based on their source of payment when providing services that

*Corresponding author. Tel: 617-432-3369; Fax: 617-432-3435; grabowski@ med.harvard.edu; Address: Department of Health Care Policy, 180 Longwood Avenue, Boston, MA 02115-5899, USA. 
are required to be provided under the law. All nursing services, specialized rehabilitative services, social services, dietary services, pharmaceutical services, or activities that are mandated by the law must be provided to residents according to residents' individual needs, as determined by assessments and care plans" (Guideline 483.12(c), Health Care Financing Administration, 1995). However, monitoring and enforcing this uniform quality constraint is quite difficult. From a behavioral perspective, there is a long-standing notion that professional norms matter in health care (Arrow, 1963). Behavioral constructs such as trust, fairness and regret may explain why providers put a value on treating all patients according to the same criteria (Frank, 2004). Finally, from an economic perspective, certain nursing home services such as dietary services are produced jointly for both payer types and may exhibit economies of joint production (Gertler and Waldman, 1992). However, the majority of direct care nursing home services would not exhibit economies of joint production. Moreover, there is some evidence from the physician and hospital settings that patients with different payer sources are treated differently (Gruber, 2000).

The assumption of common quality - and the lack of previous work analyzing this issue - also reflects the nursing home data previously available to researchers. Broadly, two alternative types of nursing home data were available: facility-level administrative databases and nationally representative surveys of nursing home residents. With the former, because the data are collected at the facility-level, there is no means by which to parse out within-facility quality differences by payer type. With the latter, the data are collected at the resident-level, but there are rarely a sufficient number of residents sampled from any one facility to make meaningful within-facility comparisons. Thus, neither of these data sources can address the research question of whether nursing home quality is a common good.

We provide the first comprehensive exploration of this question using a valuable new source of nursing home data. The Omnibus Budget Reconciliation Act of 1987 (OBRA 87) mandated that each state collect a quarterly assessment for all residents in certified nursing homes. In the fourth quarter of 1998, CMS established a national repository of these data. We have identified seven states from this national repository with accurate payer source information over time. We have access to twelve process and outcome based indicators of nursing home quality over time, allowing for a thorough analysis of quality of care.

With this unique individual level panel of nursing home data, we consider several alternative models of the relationship between payer source and quality. We begin with a cross-sectional model relating quality to payer source. Such a model suffers from two-sided selection, however: homes with more Medicaid patients may be of different quality for unobserved reasons; and patients who are on Medicaid may be differentially sick for unobserved reasons. We attempt to address both of these concerns by including fixed effects for both nursing homes, and then also for individuals. This latter model is therefore identified by the "spend down" from private-pay to Medicaid status for individual residents, and compares the change in quality over time for private-pay residents who qualify for Medicaid relative to the change in quality over time for residents who remain private payers.

Ultimately, across all three models, we find strong support for the common good assumption: quality of care is no lower for Medicaid patients within nursing homes. We assess the sensitivity of our findings to a variety of specification tests, including the use of differences in Medicaid rate differentials across states to control for selection, and find them quite robust. Thus, nursing home care does in fact appear to be a common good. 


\section{Background}

\section{Nursing Home Sector}

Nursing home residents requiring custodial care generally either pay for care privately or by qualifying for a Medicaid-financed stay. Medicaid is the dominant purchaser of nursing home services accounting for roughly $50 \%$ of all nursing home expenditures and $70 \%$ of all bed days. 5 Nursing homes are not required to admit a certain number (or proportion) of Medicaid residents. The only constraint is that a facility must be certified in order to accept Medicaid or Medicare residents (and certification is almost universal among homes with approximately $96 \%$ certified) (Strahan, 1997). ${ }^{1}$ In contrast, nursing homes are quite restricted in their ability to discharge residents (regardless of payer type) once they have been admitted. ${ }^{2}$

Private-paying residents are charged the price chosen by the home subject to market competition while Medicaid residents are paid for by various methods via state-run programs. States have considerable flexibility to design their Medicaid nursing home payment systems and set payment rates. Medicaid programs generally pay below the private price. Not surprisingly, the majority of empirical evidence supports the assertion that Medicaid residents have more restricted access to nursing homes (e.g., Ettner 1993). Medicaid eligibility is determined on a state-by-state basis by the resident's level of income and assets. Some residents qualify for Medicaid at the time of entry to the nursing home while others "spend-down" in order to qualify for coverage. That is, these individuals do not meet the state Medicaid asset threshold at the time of nursing home entry, but in the course of paying for their nursing home care, their assets decline to the state threshold and they qualify for Medicaid-financed care.

Studies have estimated that only between 10 and $25 \%$ of all private-paying residents eventually spend-down to Medicaid eligibility (Adams, Burwell and Meiners, 1993).

\section{Existing Evidence on Variation in Nursing Home Quality by Payer Type}

Chandra and Skinner (2003) suggest that disparities in health care should be decomposed into their proximate causes with respect to "across facility" variation (i.e., certain patients are admitted to facilities with poorer care practices) and "within facility" variation (i.e., certain patients are treated differently within a health care facility). A number of studies have established large cross-facility variation in health care quality. For example, in analyzing crossfacility variation in hospital quality, Hadley and colleagues (1991) compared the treatment of insured and uninsured hospital patients and found that the uninsured were $29 \%$ to $75 \%$ less likely to undergo each of five high-cost or high-discretion procedures. However, the evidence is more mixed in regards to within-facility variation. For example, Currie and Gruber (2001) found that expansions in the Medicaid program differentially affected the hospital treatment of privately insured and Medicaid insured patients. Similarly, Doyle (2005) found that the uninsured received $20 \%$ less services than the privately insured following serious motor vehicle crashes, which translated into a 1.8 percentage point higher mortality rate $($ mean $=3.8 \%)$. Alternatively, Dranove and White (1998) found that — in response to cutbacks in Medicaid and Medicare payments-service levels fell for both public and private patients, suggesting that quality may be a common good at hospitals.

\footnotetext{
${ }^{1}$ Medicaid or Medicare certification is obtained via an initial survey to verify regulatory compliance and then annually thereafter. Facilities can be certified to care for patients covered by Medicaid (15\% of all facilities), Medicare (7\%), or either payer (78\%).

${ }^{2}$ The facility may not transfer or discharge the resident unless: 1) The transfer or discharge is necessary to meet the resident's welfare and the resident's welfare cannot be met in the facility; 2) The transfer or discharge is appropriate because the resident's health has improved sufficiently so the resident no longer needs the services provided by the facility; 3) The safety of individuals in the facility is endangered; 4) The health of individuals in the facility would otherwise be endangered; 5) The resident has failed, after reasonable and appropriate notice, to pay for a stay at the facility (note that conversion from the private-pay price to payment at the Medicaid rate does not constitute non-payment); or 6) The facility ceases to operate (Health Care Financing Administration 1995).
} 
There is a substantial literature examining across-facility variation in nursing home quality by payer mix. A number of studies have shown that a higher proportion of Medicaid residents within a nursing home is negatively related to expenditures by the facility (e.g., Birnbaum et al., 1981; Nyman, 1988a; Schlenker and Shaughnessy, 1984). Gottesman (1974) found that frequency of care by staff members diminished with higher numbers of public-pay residents. In contrast, Nyman (1988c) found no significant relationship between the proportion of Medicaid residents and the ratings of patient care or dietary plans. The use of outcome measures in this literature is scant. Nyman (1988c) revealed no Medicaid-quality relationship with respect to resident care or quality-of-life measures. In related work however, Nyman (1988b) did find that regulatory violations occur with greater frequency in high Medicaid homes. In the only previous study to employ patient-level quality indicators, Mor and colleagues (2004) found that patients in "high" Medicaid (>85\%) nursing home had slightly higher physical restraint use, pressure ulcers, antipsychotic use, and pain.

There is scant research examining within-facility variation in nursing home quality. A consumer advocacy group in New York reported to the U.S. General Accounting Office (1990) that, "many nursing homes were... segregating Medicaid and private-pay residents within the nursing home" (page 18). However, there is very little work that has empirically tested this issue. Using 1983 Texas Medicaid cost report data, McKay (1989) found that the mix of Medicaid and private-pay residents did not affect a facility's marginal, average or total costs, which provides some very limited evidence that private-pay and Medicaid residents receive a uniform level of care. However, expenditures are a very indirect (and highly criticized) measure of quality. Greater expenditures can mean higher quality or they can mean increased inefficiency on the part of the facility.

Since beginning our work on this topic, a paper was published addressing cross-facility and within-facility variation in nursing home mortality. Troyer (2004) found large cross-facility differences in mortality for Medicaid and private-paying residents in Florida for the period 1986 through 1997. However, when facility fixed-effects were included, these mortality differences largely fell away, suggesting that there is not within-facility variation across Medicaid and private paying residents. Mortality is a problematic measure for this purpose, however, as it is an extreme outcome that may not be responsive to quality differences; as a result, it has not been included as a potential quality indicator in the recent validation efforts by CMS (Morris et al., 2003). In this paper, we employ a range of validated quality indicators to test our hypothesis, as well as expanding on this work to consider the role of individual fixed effects and variation in Medicaid policy across states.

\section{Data and Empirical Strategy \\ Data}

Our data are a census of all nursing home residents from seven states obtained from the Minimum Data Set (MDS) for nursing homes. Based on a federal requirement, the MDS instrument collects over 350 discrete data elements including socio-demographic information, numerous clinical items ranging from degree of functional dependence to cognitive functioning, and a checklist for staff to indicate the presence of the most common geriatric diagnoses (MDS Training Manual, 1995; Morris et al., 1994). Typically, the MDS form is filled out a by a registered nurse $(\mathrm{RN})$ working at the facility. Assessments are performed on admission, upon significant change, and at least quarterly, so that there is a panel of assessments for the same individual over time. When there were multiple assessments within a quarter, we used the assessment closest to the mid-point of the quarter.

We have access to the MDS beginning in $4^{\text {th }}$ quarter of 1998 through the $4^{\text {th }}$ quarter of 2002 (see Table 1 for descriptive statistics). As such, our data are a combination of existing residents 
(those admitted prior to $4^{\text {th }}$ quarter 1998) and new admissions (those admitted $4^{\text {th }}$ quarter of 1998 or later). We alternatively estimate models below containing the full sample $(\mathrm{N}=1,626,628)$ and only those new admissions $(\mathrm{N}=872,667)$.

The analysis was restricted to seven states because accurate payer source information was not available for the other states. Payer source on admission was nearly always recorded on the MDS form, but in many instances, the payer source field was not updated in subsequent MDS assessments. Thus, we were not able to obtain accurate payer source information over time for the majority of states. Based on a series of checks, the seven states with accurate payer source information used within this study were Kansas, Maine, Mississippi, North Dakota, Ohio, South Dakota and Washington. ${ }^{3}$

Payer status is coded on each quarterly MDS assessment based on ten categories measuring nursing home payment via (i) a Medicaid per diem, (ii) Medicare per diem, (iii) Medicare ancillary part A, (iv) Medicare ancillary part B, (v) CHAMPUS per diem, (vi) VA per diem, (vii) self or family pays full per diem, (viii) Medicaid resident liability or Medicare co-payment, (ix) private insurance per diem (including co-payment), and (x) other per diem. Importantly, these categories are not mutually exclusive. For example, in order to qualify for the Medicaid per diem payment (category i), some individuals face a co-payment (category viii) for any income they have above the state Medicaid income threshold. In an effort to create three payment categories, we used a series of decision rules in placing residents into the Medicaid, private-pay, and other payer groups. First, because we are interested in the primary payer within this study, we did not use ancillary Medicare payments (categories iii and iv) in the assignment to payer groups. Second, because we are interested in chronic (and not rehabilitative) nursing home care, we excluded those Medicare assessments from the analysis. ${ }^{4}$ That is, any assessment coded in category (ii) was dropped from our dataset. Third, any individual in category (vii) or category (ix) was considered private-pay. Fourth, we categorized all individuals from group (i) as Medicaid, regardless of whether they faced some co-payment (viii). Finally, we created an other payer group for individuals in CHAMPUS (category v), VA (category vi) and other (category $\mathrm{x}$ ).

There is no single preferred measure of nursing home quality in the MDS data, so we have formed 12 indicators of quality based on the available information (U.S. General Accounting Office, 2002). By examining patterns for all 12 indicators, we hope to assess most generally whether quality is a common good.

Our quality measures consist of five "process" based measures and seven "outcome" based measures of quality. On the surface, the outcome measures may dominate the process based measures as proxies for quality. However, there is strong evidence that the process-based measures are correlated with negative patient outcomes, and all these processes have received considerable attention from regulators, clinicians and researchers towards establishing ideal standards of care. The process based measures are:

- The presence of a physical restraint: immobility resulting from the use of physical restraints may increase the risk of pressure ulcers, depression, mental and physical deterioration, and mortality (Zinn, 1993).

\footnotetext{
${ }^{3}$ We used three separate checks to ensure the quality of the payer source field within the MDS. First, we examined whether patients that entered under Medicare converted to another payer type at the 100-day Medicare limit. Second, we compared the aggregate facility-level payer mix from the MDS with the aggregate payer mix from the Federal government's Online, Survey Certification and Reporting System. Finally, we compared spend-down rates from private-pay to Medicaid status in the MDS with published estimates from other data sources.

${ }^{4}$ Our results are robust to including post-acute Medicare patients in the analysis.
} 
- Use of an indwelling catheter: urethral catheterization places the resident at greater risk for urinary tract infection; other long-term complications include bladder and renal stones, abcesses, and renal failure.

- Bedfast: although many residents are bedfast due to medical conditions, bedfast residents are at a higher risk of developing pressure ulcers and other complications.

- Use of a feeding tube: feeding tubes can result in complications including selfextubation, infections, aspiration, unintended misplacement of the tube, and pain.

- The use of anti-psychotics: overuse of anti-psychotics may result in mental and physical deterioration (Harrington et al, 1992).

The outcome based measures are:

- Pressure ulcers: pressure ulcers are areas of the skin and underlying tissues that erode as a result of pressure or friction and/or lack of blood supply.

- The presence of daily pain: pain is a common condition related to numerous age associated diseases such as cancer, arthritis, and diabetic neuropathy. However, pain can be moderated, and the management of pain in the nursing home setting is often substandard (Ferrell, 1995).

- Bowel or bladder incontinence: although some decline in bladder or bowel continence may not be reversible or manageable in the latter stages of disease (e.g., dementia, terminal illness), it is thought to be preventable and reversible in the nursing home population (Schnelle and Leung, 2004).

- Urinary tract infections and wound infections: infections occur frequently among nursing home residents with the potential for significant morbidity and mortality (Nicolle, 2000).

- Presence of a fall: over the 30 days prior to the assessment: falls are a major source of morbidity and mortality among nursing home residents. Although falls have been shown to be preventable in the nursing home environment, approximately half of all patients fall each year, and 9\% sustain serious injury (Ray et al., 1997).

- Depression: major depression is associated with increased functional dependence, health care utilization, and mortality (Simmons et al., 2004).

A number of patient-level covariates were available from the MDS. In order to capture the need for services, an activities of daily living (ADL) score was created for each resident assessment. The ADL score is based on the individual's need for help with bathing, bed mobility, dressing, eating, toileting, and transferring. For each of these six areas, the individual is scored as a zero (independent), one (supervised), two (limited assistance), three (extensive assistance) or four (total dependence). Thus, the total ADL score ranges between zero and twenty-four. For the analyses, we included 24 dummy variables in our regressions. We also included dummy variables for the Cognitive Performance Scale (CPS), ranging from zero to six in value (Morris et al 1994).

We also include a set of dummy variables measuring length-of-stay. With these dummy variables in our regressions, it is important to note that we are not simply identifying length of stay effects, but true transition effects. Specifically, we created a dummy variable to approximate length of stay for each quarter up through 10 years in the nursing home. The final dummy variable measures length of stay greater than 10 years. Thus, we have 40 dummy variables to model length of stay. Finally, we also included a number of demographic variables including age, gender, race, education, and marital status. 
The MDS assessments from these seven states were merged with two other facility-level data sources. First, the MDS is a resident-level instrument that does not contain facility-level information. Thus, ownership status and other institutional information on nursing homes are obtained from the On-Line Survey, Certification, and Reporting (OSCAR) system. The OSCAR system contains information from state surveys of all federally certified Medicaid (nursing facilities) and Medicare (skilled nursing care) homes in the United States. Certified homes represent almost 96\% of all facilities nationwide (Strahan, 1997). Collected and maintained by the Centers for Medicare and Medicaid Systems (CMS), the OSCAR data are used to determine whether homes are in compliance with federal regulatory requirements. Every facility is required to have an initial survey to verify compliance. Thereafter, states are required to survey each facility no less often than every 15 months, and the average is about 12 months (Harrington et al., 1999). From the OSCAR, we included measures of bed size, ownership status, and chain ownership.

Second, we obtained nursing home cost report information from each state's Medicaid office. These annual cost reports provided data on the private-pay and Medicaid payment rates for each facility. Table 2 provides the average payment rates for each state and year contained in our study. Without adjusting for resident acuity, the Medicaid payment rate ranges from $76 \%$ to $108 \%$ of the private-pay price. North Dakota is the only state with a higher (unadjusted) Medicaid rate relative to the private-pay rate across all years. ${ }^{5}$ North Dakota has a rate equalization law under which all residents - regardless of payer type - are grouped into one of thirty-five payment levels based on their acuity. Thus, the higher aggregate Medicaid payment rate reflects the fact that Medicaid patients are less healthy relative to their privatepay counterparts.

\section{Empirical Strategy}

We pursue a variety of empirical specifications designed to determine whether Medicaid patients are treated in a systematically different fashion than non-Medicaid patients. We begin by estimating cross-sectional models of the form:

$$
\mathrm{Y}_{\mathrm{int}}=\alpha+\beta_{1} \mathrm{OTHER}_{\mathrm{int}}+\beta_{2} \text { MEDICAID }_{\mathrm{int}}+\delta \mathrm{X}_{\mathrm{int}}+\gamma \mathrm{Z}_{\mathrm{nt}}+\alpha_{\mathrm{t}}+\varepsilon_{\mathrm{int}}
$$

where $\mathrm{i}$ indexes individuals, $\mathrm{n}$ indexes nursing homes, and $\mathrm{t}$ indexes time periods, $\mathrm{Y}$ is the outcome variable, OTHER and MEDICAID are dummies for other payer and Medicaid (relative to private-pay), $\mathrm{X}$ is a set of individual characteristics, $\mathrm{Z}$ is a set of nursing home characteristics, $\alpha_{t}$ are time (quarter) fixed effects, and $\varepsilon_{\text {int }}$ is the randomly distributed error term. The quarter dummies control for national trends in nursing home quality that may be correlated with shifts in the mix of Medicaid and private-pay residents.

As noted in the introduction, models such as (1) will be biased due to both unobserved facility and individual characteristics. Our second specification deals with the first of these problems by including nursing home fixed effects $\left(\lambda_{n}\right)$ :

$\mathrm{Y}_{\mathrm{int}}=\alpha+\beta_{1}$ OTHER $_{\text {int }}+\beta_{2}$ MEDICAID $_{\text {int }}+\delta \mathrm{X}_{\mathrm{int}}+\gamma \mathrm{Z}_{\mathrm{nt}}+\alpha_{\mathrm{t}}+\lambda_{\mathrm{n}}+\varepsilon_{\mathrm{int}}$

The nursing home fixed effects control for any fixed facility-specific omitted variables correlated with the quality of care such as the facility's management philosophy or facility culture. Thus, Equation (2) deals with the first type of selection identified above, that homes with a higher share of Medicaid may be ones that treat their patients worse for other reasons.

\footnotetext{
${ }^{5}$ The results presented in this paper are robust to excluding the North Dakota assessments from the analyses.
} 
Although this fixed-effects specification eliminates time-invariant heterogeneity at the level of the facility, it may still suffer from bias due to unobserved heterogeneity at the level of the patient. For example, even though we control for detailed case-mix information in the model above, there may still exist unobserved health characteristics that influence both nursing home choice and outcomes. Thus, we next estimate models of the following form:

$\mathrm{Y}_{\text {int }}=\alpha+\beta_{1}$ OTHER $_{\text {int }}+\beta_{2}$ MEDICAID $_{\text {int }}+\delta \mathrm{X}_{\text {int }}+\gamma \mathrm{Z}_{\mathrm{nt}}+\alpha_{\mathrm{t}}+\mu_{\mathrm{i}}+\varepsilon_{\text {int }}$

where the model specification is identical to equation 2 above except the facility fixed effects are replaced with patient fixed effects $\mu_{\mathrm{i}}$. In this model, the basic identification strategy purges the unobserved time-invariant heterogeneity by relying on spend-down from private-pay to Medicaid status over time. ${ }^{6}$ That is, this model controls for fixed but unobservable characteristics of both nursing homes and patients, allowing us to ask: does treatment quality change when patients change payer source?

There are two potential problems with this empirical framework, however. The first is the potential endogeneity of payer status and quality. There may exist unobserved, time-varying factors that are correlated with both payer status and quality, for example if somehow negative health shocks lead individuals to switch to Medicaid. We address this concern in two ways below. First, we explicitly model observable patient health as a function of payer status, to see if there is a correlation with a measurable source of selection. We find that, once patient fixed effects are included, such correlation is quite small. Second, we provide estimates that exploit cross-state variation in the difference between Medicaid payment rates and private-pay prices (see Table 2). In both the facility (eq. 2) and individual (eq. 3) fixed effects models, we incorporate both a fee ratio, which is the average state Medicaid payment rate divided by the average state private-pay price, and an interaction term between this fee ratio and Medicaid status. If Medicaid residents receive lower quality than their private-pay counterparts, this effect should be largest in states with the greatest difference between the private-pay price and the Medicaid payment rate; where this fee differential is zero, there should be no quality difference. This model is free of selection as long as any bias from time-varying unobserved variables is equally important in high and low differential homes/states.

The other problem with equation (3) is that it captures a specific type of payer source change, the spend-down from private pay to Medicaid. It is possible that patients who are admitted from the start as Medicaid are treated worse than those who are private pay, but that when private pay patients transition to Medicaid their treatment does not change (for example because the nursing home has already established a treatment regime). That is, by identifying our models from transitions, we may miss important elements of nursing home discrimination that apply only to those admitted under different payer statuses, not those who transition across payer status.

There is no way to both address this problem and control for unobserved patient characteristics. However, we can determine the magnitude this problem by focusing solely on new nursing home admissions (i.e., individuals admitted in the $4^{\text {th }}$ quarter of 1998 or later), and estimating the model as a function of payer status at admission. We present results from this approach below.

Given the size of our dataset, least squares models estimations of linear probability regression models are presented. Although this approach does not recognize the binary nature of the

\footnotetext{
${ }^{6}$ Person fixed effects will subsume home fixed effects for patients who do not move between homes. However, the overall transfer rate per year at risk has been estimated at 3.3\% (Hirth et al, 2003), so this is not a large issue.
} 
quality measures, it facilitates the tractable estimation of these models, which are based on a large number of observations and an expansive set of regression controls. Using a sub-sample of the data however, the linear probability estimates were robust to those obtained using a conditional logit model. In the results tables presented below, only the Medicaid coefficients and the absolute value of the t-statistics are included. Because we have multiple observations on each individual, standard errors are clustered at the level of the individual.

\section{Basic Results}

The first set of regression results includes both the existing residents when our MDS panel started and residents admitted in that and subsequent quarters (see Table 3). Importantly, all twelve outcomes are negative indicators of quality; thus, a positive Medicaid coefficient translates into lower quality for this payer group. Moreover, given our huge sample size, it should be noted that we have a great deal of precision in our estimates. Thus, nearly every coefficient estimate presented in Table 3 is statistically significant at the conventional level.

The first column of results excludes fixed-effects and is identified solely by cross-sectional variation in payer source and quality. The first five rows present the process-based quality measures; the next seven rows show the outcome-based quality measures.

The results from this cross-sectional model are surprisingly mixed: contrary to expectations, we do not find clear evidence of lower quality for Medicaid patients, even without controlling for home or person fixed effects. For the process measures, three are positive, indicating worse quality for Medicaid patients (restraints, anti-psychotics, and feeding tubes), although only one (anti-psychotics) is significant; two are negative, indicating better quality for Medicaid patients (catheters, bedfast), and both are significant. For the outcome measures, three are positive, indicating worse quality for Medicaid patients (pain, incontinence, and depression), while four are negative, indicating better quality for Medicaid patients (pressure ulcers, urinary infection, wound infection, and falls); all are significant. These mixed findings are surprising given the general presumption that Medicaid patients are treated worse in nursing homes. ${ }^{7}$

The effects, however, are uniformly very small. The only effects that amount to more than $10 \%$ of the baseline means in Table 1 are catheters $(-11 \%)$, wound infections $(-20 \%)$, and falls $(-14 \%)$. Given this pattern, the weight of the evidence actually appears to point towards better quality for Medicaid patients. But the overall pattern is one of small and inconsistent effects.

The second column presents results from a model that includes nursing home fixed effects. This model is identified by within-facility variation in payer status and nursing home quality, so that we are comparing the treatment of Medicaid and non-Medicaid patients within the same facility. The results are remarkably similar to those without home fixed effects. Indeed, if anything, there is even more evidence of a positive treatment of Medicaid patients in this model, as the two process measures which were insignificant and positive (restraints and feeding tubes) are now negative and (at least marginally) significant. But the results remain small overall, and once again mixed, with positive coefficients (negative quality) on important measures such as pain, incontinence, and depression.

\footnotetext{
${ }^{7}$ Although the empirical literature has generally argued that quality decreases as the proportion of Medicaid patients increases, these studies have typically employed problematic quality measures such as staffing or regulatory deficiencies. The only other study to examine this relationship using patient level MDS quality indicators found that "high" Medicaid (>85\%) facilities had slightly higher restraints, pressure ulcers, antipsychotic use, and pain relative to all other facilities (Mor et al, 2004). As a robustness check, we analyzed whether "high" Medicaid homes provided worse quality. The restraint, anti-psychotic, feeding tube and incontinence measures were all higher in these facilities, but other measures did not indicate worse Medicaid quality.
} 
The third column presents results from a model including patient-level fixed effects, which is identified by within-patient variation (i.e., spend-down to Medicaid). These results have shifted slightly from the results including home fixed effects, but they present a similar story: mixed evidence that, if anything, suggests higher quality for Medicaid patients. Once again, the results remain small in magnitude. The estimates that amounted to more than $10 \%$ of their baseline values were catheters $(-15 \%)$, feeding tubes $(-10 \%)$, pressure ulcers $(-12 \%)$, urinary infections $(-14 \%)$, wound infections $(-20 \%)$ and falls $(-12 \%)$. Put alternatively, the estimated coefficients in column 3 were each less than $6 \%$ of a standard deviation of the dependent variable means. ${ }^{8}$

In sum, the fixed effects specifications provide inconsistent results as to whether nursing home quality is a common good. Some measures indicated worse quality for Medicaid recipients while others indicated better quality. In general, the magnitude of the coefficients was quite small.

\section{Addressing Selection and Locality of Treatment Effects}

The inclusion of both nursing home and patient-level fixed effects in our models should, in principle, address selection problems. However, it remains possible that patients are becoming unobservably sicker, or even healthier, around the time of transition to Medicaid. This could bias our results in the direction of finding negative, or positive, effects of Medicaid on quality. In addition, as discussed earlier, our models with patient fixed effects are identified only from transitions, and it is possible that there is differential treatment of Medicaid patients upon arrival that is not present when they transition. This is the problem of "local average treatment effects", whereby our estimates reflect the true answer for a particular population but not for others.

We address these two issues in this section of the paper. First, we assess whether selection appears to be a problem by examining observable indicators of health to see if they are correlated with transitions to Medicaid payer status. We then turn to using rate differentials across states to add extra identification to the model. Finally, we consider models that focus just on payer status at admission to assess whether there is differential treatment based on initial payer.

\section{Selection on Observables}

The inclusion of fixed effects in our models is designed to capture home and individual-based selection that might bias our quality estimates. The robustness of our results across the two alternate identification strategies is reassuring, but we can get some perspective on this issue by looking at observable correlates of selection. As argued formally by Altonji and colleagues (2000), if observables are sufficiently predictable of unobservables, then assessing the correlation of the variable of interest with observables can provide some indicator of the potential bias from omitting unobservables. In our context, the unobservable of most interest is health, and we have two excellent observable indicators of health: scales measuring physical and cognitive performance.

We therefore re-estimate each of our models where the dependent variable, quality, is alternately replaced with two measures of observable health, the ADL index described earlier, which takes on values of $0-24$ and the CPS score, which takes on values of $0-6$. We then assess whether our Medicaid indicator is correlated with the health measure in each of our

\footnotetext{
${ }^{8}$ As an additional check, we also estimated the cross-facility (column 2) and cross-person (column 3) variance associated with the estimated fixed effect. Using the facility fixed effects, six of the twelve estimated coefficients exceeded $10 \%$ of a standard deviation of the estimated fixed effect. However, in the person fixed effect model, none of the estimated coefficients exceeded 6\%. By comparison, other variables in the person fixed effects model, such as the ADL dummies, implied large effects relative to this same benchmark.
} 
specifications, and whether the correlation is substantively large enough to likely bias our estimates.

The results of this exercise are shown in Table 4. For models without home/person fixed effects, Medicaid status is positively correlated with the ADL score and negatively correlated with the CPS score, implying Medicaid patients are in better physical health, but worse cognitive health. The results show that being on Medicaid is associated with a reduction in ADL score of 0.384 , relative to a mean score of 14.4 , and an increase in CPS score of 0.063 , relative to a mean score of 2.92. This suggests that, if anything, our results without home/person fixed effects are biased against finding low quality along "physical" dimensions for Medicaid patients, but biased in favor of finding low quality along cognitive dimensions.

When we include nursing home fixed effects in the second column, the ADL coefficient does not change much: there remains a strong negative correlation with the ADL score (actually even stronger than in the first column). However, there is a small negative correlation between the CPS score in this specification. The results for both measures change significantly in the third column. When person fixed effects are included: there is now a small positive correlation with Medicaid. That is, this model indicates that individuals are, along observable dimensions, becoming sicker when they transition to Medicaid. This is indicative of selection which biases in favor of finding low quality for Medicaid patients. The effect is very small, however, and amounts to only $0.5 \%$ (ADL) and $1.3 \%$ (CPS) of the sample mean; they are only significant because of the large size of our sample. Thus, any selection effects based on observables are quantitatively small, and work towards finding lower quality for Medicaid patients. This does not rule out selection on unobservables, but it does suggest such selection is not importantly biasing us towards finding higher quality for Medicaid patients.

\section{Rate Differential Results}

As noted in Table 2, there is some variation across states in the Medicaid rate-private pay price differential. Given potential concerns with the endogeneity of payer status and quality, we used the payment differential as an exogenous source of variation across states. Thus, we tested whether quality differences between private-pay and Medicaid residents were greatest in those states with the largest differential between the private-pay price and Medicaid rate. The key coefficient of interest is the interaction of a state's Medicaid-to-private rate ratio with an individual's Medicaid status. If lower Medicaid reimbursement is leading to worse treatment of Medicaid patients, then as the rate differential increases towards one (i.e., equal payment for private-pay and Medicaid), we would expect less difference in quality across Medicaid and private-pay patients (thus, negative coefficient estimates).

Table 5 presents the coefficients of interest from this specification for our patient fixed effects model. There is very little support for the idea that quality is worse in those states with a greater payment differential. In fact, most of the estimated coefficients indicate the opposite effect. Only four of the fee interaction coefficients are negative, and only those for restraints, incontinence and depression are significant. Strikingly, these are the same outcomes for which the main effects on Medicaid status are positive: that is, for these outcomes, quality worsens when individuals transition to Medicaid, and less so when the Medicaid payment differential is smaller. Thus, it is possible that there is some effect of higher Medicaid rates in improving outcomes in these areas. Overall, however, the pattern does not indicate that increased Medicaid rates (relative to private-pay prices) lead to better outcomes, consistent with our earlier 
findings. ${ }^{9}$ Given the range of different outcomes examined in this table, this evidence is fairly weak in support of quality as a private good.

\section{Local Treatment Effects}

The other major concern discussed earlier was that, although including person fixed effects deals with selection problems in these regressions, it captures only a particular source of the variation in treatment, that due only to treatment changes as individuals transition to Medicaid. It is possible that there could be no change in treatment when individuals make such a transition, while still being discriminatory in the treatment of patients who arrive as Medicaid. That is, the local treatment effect we estimate from this group of switchers may not represent the true effects of Medicaid status on treatment. ${ }^{10}$

To address this concern, we replace our measure of payer status with a measure based on initial payer status on admission (see Table 6). That is, we are not comparing those who changed status in these models, but rather comparing individuals based on what they were labeled when they entered the home. Obviously, in this case, we cannot include person-specific fixed effects, so Table 6 shows only models with no fixed effects and home-specific fixed effects.

In fact, the results are strikingly similar to our earlier findings: by and large positive association between Medicaid and quality (negative coefficients), with some negative effects (positive coefficients) for incontinence, anti-psychotics, and depression. Once again, all of the coefficients are very small, suggesting no effect in the aggregate even when the model is identified based on those who switch payer status.

\section{Conclusions}

Using a variety of identification strategies, the results in this paper confirm the common presumption that nursing home quality is a common good. Specifically, we employed models identified by within-facility and within-person variation in payer status and quality; we showed that selection on observable dimensions is small; we exploited cross-state variation in the Medicaid and private-pay rate differential as an additional source of variation; and we considered individuals solely based on payer status at admission. In every case, there was no consistent finding of differential quality for Medicaid patients, and the coefficients of either sign were universally quite small. Overall, our results are consistent with the assumption of uniform quality across Medicaid and private-paying residents within facilities.

This result has both research and policy implications. From a research perspective, this study provides support for the long-standing practice of using facility-level data to evaluate Medicaid policy effects in the nursing home literature (e.g., Gertler, 1989). Although the MDS and other individual-level data are now becoming available to researchers, aggregate facility-level measures can still serve as a potential complement to the individual-level measures. Although there is evidence that quality is often a private good in the physician and hospital sectors, researchers have also found support for the common good assumption across publicly and privately insured patients in hospitals (Dranove and White, 1998).

\footnotetext{
${ }^{9}$ In a similar robustness check, we also analyzed whether commonality in quality differed across nonprofit and for-profit nursing homes. When we interacted the Medicaid dummy with the ownership type of the facility, we found that commonality in quality was consistent across nonprofit and for-profit nursing homes (results available upon request from the authors).

${ }^{10} \mathrm{~A}$ related concern at the facility-level is that differential Medicaid quality may be a function of the underlying proportion of Medicaid patients within the nursing homes. For example, high Medicaid homes may treat everyone similarly, but low Medicaid homes may differentiate between Medicaid and private-pay patients. Using local linear regression, we did not find large treatment differences across high and low Medicaid homes in their treatment of private-pay and Medicaid patients.
} 
From a policy perspective, these results point towards the potential for free-ridership on the part of state Medicaid programs. If quality is a common good enjoyed equally by all payer types, then state Medicaid programs can potentially free ride on higher paying private residents. Although state Medicaid administrators have historically used certificate-of-need laws to help keep private-paying residents in joint care settings, two recent trends potentially undermine the value of private-paying patients to state Medicaid nursing home programs. First, assisted living and other nursing home substitutes have siphoned off some private-paying residents. In many instances, individuals exhaust their private resources in the assisted living sector before transitioning to Medicaid-financed nursing home care. Second, the nursing home industry has become more segregated across facilities over the last several years (Mor et al., 2004). That is, there are more facilities caring for predominantly Medicaid (or private-pay) residents, implying less potential for cross-subsidization across payer types. Thus, although quality may be uniform within facilities, market-based trends point towards fewer private-paying residents going to homes with Medicaid patients.

\section{Acknowledgments}

We are grateful to the National Institute on Aging (K01 AG024403), the Centers for Medicare \& Medicaid Services, and the National Bureau of Economic Research for providing funding for this study. Data were analyzed under CMS data use agreement number 15989. We thank Lee Howard and Xinzhi Zhang for excellent research assistance on this project and Tom McGuire, Neeraj Sood and Aaron Yelowitz for helpful comments.

\section{References}

Adams, E Kathleen; Meiners, Mark R.; Burwell, Brian O. Asset Spend-Down in Nursing Homes. Methods and Insights. Medical Care 1993;31(1):1-23. [PubMed: 8417267]

Altonji, Joseph G.; Elder, Todd E.; Taber, Christopher R. Selection on Observed and Unobserved Variables: Assessing the Effectiveness of Catholic Schools. National Bureau of Economic Research. 2000 Working Paper No. 7831.

Arrow, Kenneth J. Uncertainty and the Welfare Economics of Medical Care. American Economic Review 1963;53(5):941-973.

Chandra, Amitabh; Skinner, Jonathan. Geography and Racial Health Disparities. National Bureau of Economic Research. 2003 Working Paper No. 9513.

Currie, Janet; Gruber, Jonathan. Public Health Insurance and Medical Treatment: The Equalizing Impact of the Medicaid Expansions. Journal of Public Economics 2001;82(1):63-89.

Doyle, Joseph J. Health Insurance, Treatment, and Outcomes: Using Auto Accidents as Health Shocks. Review of Economics and Statistics 2005;87(2):256-270.

Dranove, David; White, William D. Medicaid-dependent hospitals and their patients: how have they fared? Health Services Research 1998;33(2 Pt 1):163-185. [PubMed: 9618666]

Frank, Richard G. Behavioral Economics and Health Economics. (2004). National Bureau of Economic Research. 2004 Working Paper No. 10881.

Freiman, Marc P.; Murtaugh, Christopher M. The determinants of the hospitalization of nursing home residents. Journal of Health Economics 1993;12(3):349-359. [PubMed: 10129842]

Gertler, Paul J. Subsidies, quality, and the regulation of nursing homes. Journal of Public Economics 1989;38(1):33-52.

Gertler, Paul J.; Waldman, Donald M. Quality-Adjusted Cost Functions and Policy Evaluation in the Nursing Home Industry. Journal of Political Economy 1992;100(6):1232-1256.

Grabowski, David C. Medicaid Reimbursement and the Quality of Nursing Home Care. Journal of Health Economics 2001;20(4):549-569. [PubMed: 11463188]

Grabowski, David C. A Longitudinal Study of Medicaid Payment, Private-Pay Price and Nursing Home Quality. International Journal of Health Care Finance and Economics 2004;4(1):5-26. [PubMed: 15170962]

Gruber, Jonathan. Medicaid. National Bureau of Economic Research. 2000 Working Paper No. 7829. 
Hadley, Jack; Steinberg, Earl P.; Feder, Judith. Comparison of uninsured and privately insured hospital patients. Condition on admission, resource use, and outcome. JAMA 1991;265(3):374-379. [PubMed: 1984537]

Health Care Financing Administration. State Operations Manual: Provider Certification. Department of Health and Human Services. 1995 June; HCFA Pub. 7, Transmittal No. 274.

Hirth, Richard A.; Banaszak-Holl, Jane C.; Fries, Brant E.; Turenne, Marc N. Does quality influence consumer choice of nursing homes? Evidence from nursing home to nursing home transfers. Inquiry 2003;40(4):343-361. [PubMed: 15055834]

Jacobson, Louis S.; Lalonde, Robert J.; Sullivan, Daniel G. Earnings Losses of Displaced Workers. American Economic Review 1993;83(4):685-709.

McKay, Nicole L. Quality Choice in Medicaid Markets: The Case of Nursing Homes. Quarterly Review of Economics and Business 1989;29:27-40. [PubMed: 10303971]

Mor, Vincent; Zinn, Jacqueline; Angelelli, Joseph; Teno, Joan M.; Miller, Susan C. Driven to tiers: socioeconomic and racial disparities in the quality of nursing home care. Milbank Quarterly 2004;82 (2):227-256. [PubMed: 15225329]

Morris, John N.; Fries, Brant E.; Mehr, David R.; Hawes, Catherine; Phillips, Charles; Mor, Vincent; Lipsitz, Lewis A. MDS Cognitive Performance Scale. Journal of Gerontology 1994;49(4):M174M182. [PubMed: 8014392]

Morris, John N.; Moore, Terry; Jones, Rich; Mor, Vincent; Angelelli, Joseph; Berg, Katherine; Hale, Catherine; Morris, Shirley; Murphy, Katharine M.; Rennison, Melissa. Validation of Long-Term and Post-Acute Quality Indicators. Baltimore, MD: Centers for Medicare and Medicaid Services; 2003.

Nicolle, Lindsay E. Infection Control in Long-term Care Facilities. Clinical Infectious Diseases 2000;31 (3):752-756. [PubMed: 11017825]

Norton, Edward C. Long-term Care. In: Cuyler, AJ.; Newhouse, JP., editors. Handbook of Health Economics. Amsterdam: Elsevier Science; 2000. p. 955-994.

Nyman, John A. The effect of competition on nursing home expenditures under prospective reimbursement. Health Services Research 1988a;23(4):555-574. [PubMed: 3141316]

Nyman, John A. Excess demand, the percentage of Medicaid patients, and the quality of nursing home care. Journal of Human Resources 1988b;23(1):76-92.

Nyman, John A. Improving the quality of nursing home outcomes. Are adequacy- or incentive-oriented policies more effective? Medical Care 1988c;26(12):1158-1171. [PubMed: 3199912]

Ray, Wayne A.; Taylor, Jo A.; Meador, Keith G.; Thapa, Purushottam B.; Brown, Anne K.; Kajihara, Henry K.; Davis, Claudia; Gideon, Patricia; Griffin, Marie R. A Randomized Trial of a Consultation Service to Reduce Falls in Nursing Homes. JAMA 1997;278(7):557-562. [PubMed: 9268276]

Scanlon, William J. A theory of the nursing home market. Inquiry 1980;17(1):25-41. [PubMed: 6445331]

Schnelle, John F.; Leung, Felix W. Urinary and fecal incontinence in nursing homes. Gastroenterology 2004;126(1 Suppl 1):S41-S47. [PubMed: 14978637]

Simmons SF, Cadogan MP, Cabrera GR, Al-Samarrai NR, Jorge JS, Levy-Storms L, Osterwei D, Schnelle JF. The minimum data set depression quality indicator: does it reflect differences in care processes? Gerontologist 2004;44(4):554-564. [PubMed: 15331813]

Troyer, Jennifer L. Examining differences in death rates for Medicaid and non-Medicaid nursing home residents. Medical Care 2004;42(10):985-991. [PubMed: 15377931]

U.S. General Accounting Office. Nursing Homes: Admission Problems for Medicaid Recipients and Attempts to Solve Them. Washington, D.C: 1990. Pub. No. HRD-90-135

U.S. General Accounting Office. Nursing Homes: Public Reporting of Quality Indicators Has Merit, but National Implementation Is Premature. Washington, D.C: 2002. Pub. No. GAO-03-187

Weissert, William C.; Scanlon, William J. Determinants of nursing home discharge status. Medical Care 1985;23(4):333-343. [PubMed: 3887051] 
Table 1

Summary Statistics

\begin{tabular}{|c|c|c|c|c|}
\hline & \multicolumn{2}{|c|}{ All assessments } & \multicolumn{2}{|c|}{ Observed from admission } \\
\hline & Mean & SD & Mean & SD \\
\hline \multicolumn{5}{|l|}{ Quality Indicators } \\
\hline Physical Restraints & 0.09 & 0.28 & 0.07 & 0.26 \\
\hline Catheters & 0.08 & 0.26 & 0.09 & 0.29 \\
\hline Bedfast & 0.06 & 0.23 & 0.05 & 0.22 \\
\hline Feeding Tubes & 0.07 & 0.25 & 0.06 & 0.24 \\
\hline Anti-psychotics & 0.19 & 0.39 & 0.21 & 0.41 \\
\hline Pressure Ulcers & 0.08 & 0.27 & 0.09 & 0.28 \\
\hline Pain & 0.13 & 0.34 & 0.14 & 0.35 \\
\hline Incontinence & 0.54 & 0.50 & 0.46 & 0.50 \\
\hline Urinary infection & 0.09 & 0.29 & 0.11 & 0.31 \\
\hline Wound infection & 0.02 & 0.13 & 0.02 & 0.15 \\
\hline Fall & 0.16 & 0.37 & 0.20 & 0.40 \\
\hline Depression & 0.43 & 0.50 & 0.42 & 0.49 \\
\hline \multicolumn{5}{|l|}{ Payer Source } \\
\hline Medicaid & 0.65 & 0.48 & 0.55 & 0.50 \\
\hline Private-payer & 0.32 & 0.47 & 0.40 & 0.49 \\
\hline Other payer & 0.04 & 0.19 & 0.04 & 0.20 \\
\hline \multicolumn{5}{|l|}{ Demographics } \\
\hline Female & 0.71 & 0.46 & 0.68 & 0.47 \\
\hline Age & 80.48 & 12.86 & 79.37 & 13.11 \\
\hline African American & 0.09 & 0.29 & 0.09 & 0.29 \\
\hline Caucasian & 0.90 & 0.30 & 0.90 & 0.30 \\
\hline Other Race & 0.01 & 0.11 & 0.01 & 0.12 \\
\hline No High School & 0.18 & 0.38 & 0.16 & 0.37 \\
\hline High School & 0.17 & 0.37 & 0.18 & 0.38 \\
\hline Some College & 0.06 & 0.24 & 0.07 & 0.25 \\
\hline College & 0.03 & 0.18 & 0.04 & 0.19 \\
\hline Education Missing & 0.56 & 0.50 & 0.56 & 0.50 \\
\hline Married & 0.19 & 0.39 & 0.23 & 0.42 \\
\hline Never Married & 0.13 & 0.34 & 0.12 & 0.32 \\
\hline Widowed & 0.57 & 0.49 & 0.55 & 0.50 \\
\hline Divorced/Separated & 0.11 & 0.31 & 0.11 & 0.31 \\
\hline \multicolumn{5}{|l|}{ Health/Length of Stay } \\
\hline ADL Score (0-24) & 14.04 & 6.73 & 13.15 & 6.41 \\
\hline CPS Score (0-6) & 2.92 & 1.79 & 2.61 & 1.73 \\
\hline Length of stay (days) & 850.23 & $1,146.30$ & 289.96 & 307.57 \\
\hline \multicolumn{5}{|l|}{ Facility Characteristics } \\
\hline Beds & 130.02 & 76.94 & 130.43 & 81.92 \\
\hline
\end{tabular}

Rev Econ Stat. Author manuscript; available in PMC 2010 May 11. 


\begin{tabular}{lccccc}
\hline & \multicolumn{2}{c}{ All assessments } & \multicolumn{2}{c}{ Observed from admission } \\
\cline { 2 - 3 } & Mean & SD & & Mean & SD \\
\cline { 2 - 3 } \cline { 5 - 6 } For-profit & 0.69 & 0.46 & & 0.69 & 0.46 \\
Nonprofit & 0.28 & 0.45 & & 0.28 & 0.45 \\
Government & 0.03 & 0.16 & & 0.02 & 0.15 \\
Chain Facility & 0.62 & 0.49 & & 0.63 & 0.48 \\
Urban Facility & 0.69 & 0.46 & & 0.71 & 0.45 \\
State & & & & 0.07 & 0.25 \\
Kansas & 0.08 & 0.27 & & 0.07 & 0.25 \\
Maine & 0.07 & 0.25 & & 0.07 & 0.21 \\
Mississippi & 0.05 & 0.21 & & 0.04 & 0.22 \\
North Dakota & 0.06 & 0.23 & & 0.05 & 0.49 \\
Ohio & 0.59 & 0.49 & & 0.58 & 0.15 \\
South Dakota & 0.03 & 0.16 & & 0.02 & 0.37 \\
Washington & 0.14 & 0.35 & 0.17 & $0.372,667$ \\
N & $1,626,628$ & &
\end{tabular}

Notes: There are missing data for certain variables. $\mathrm{ADL}=$ activities of daily living; $\mathrm{CPS}=$ cognitive performance scale. 


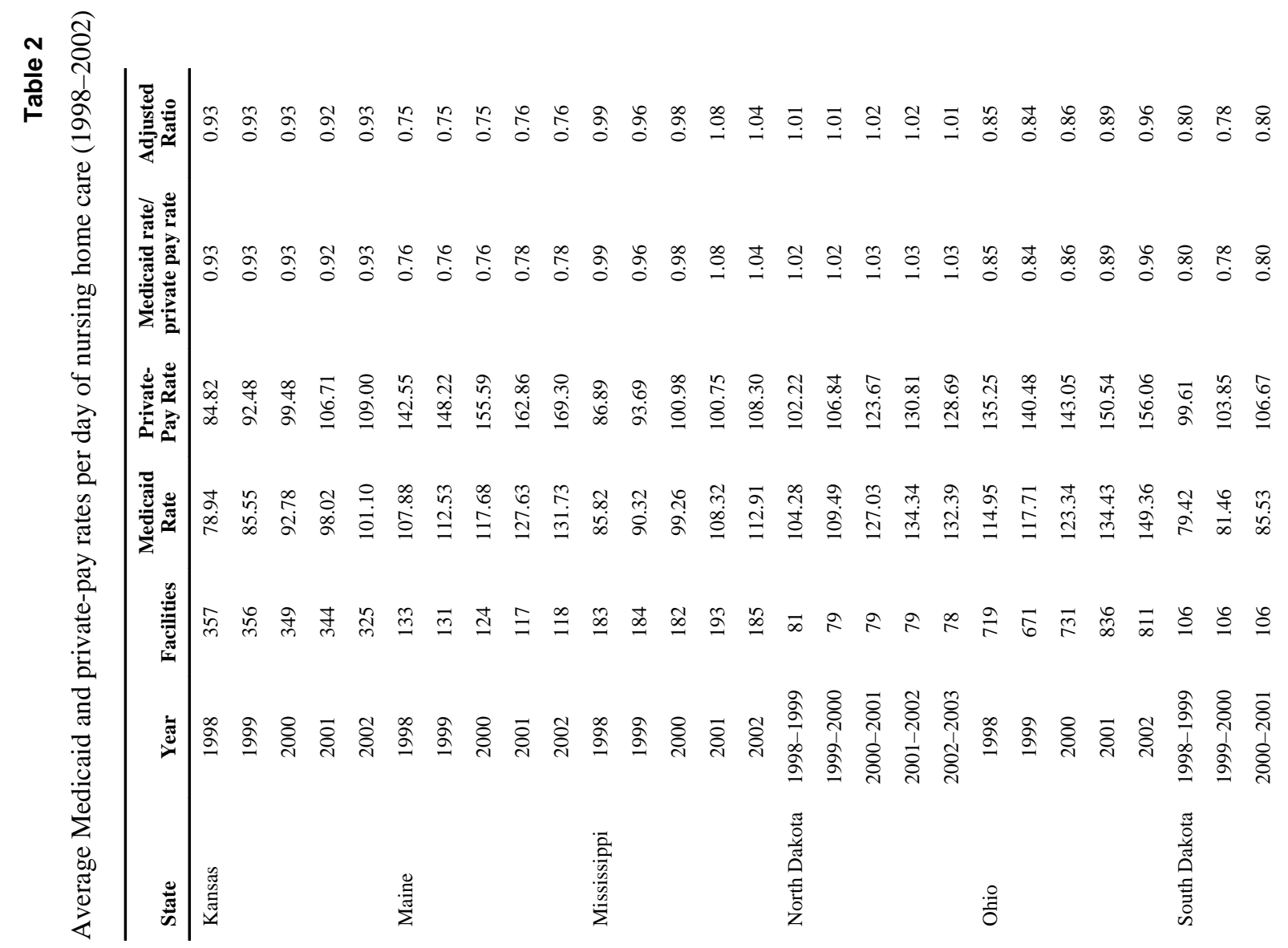




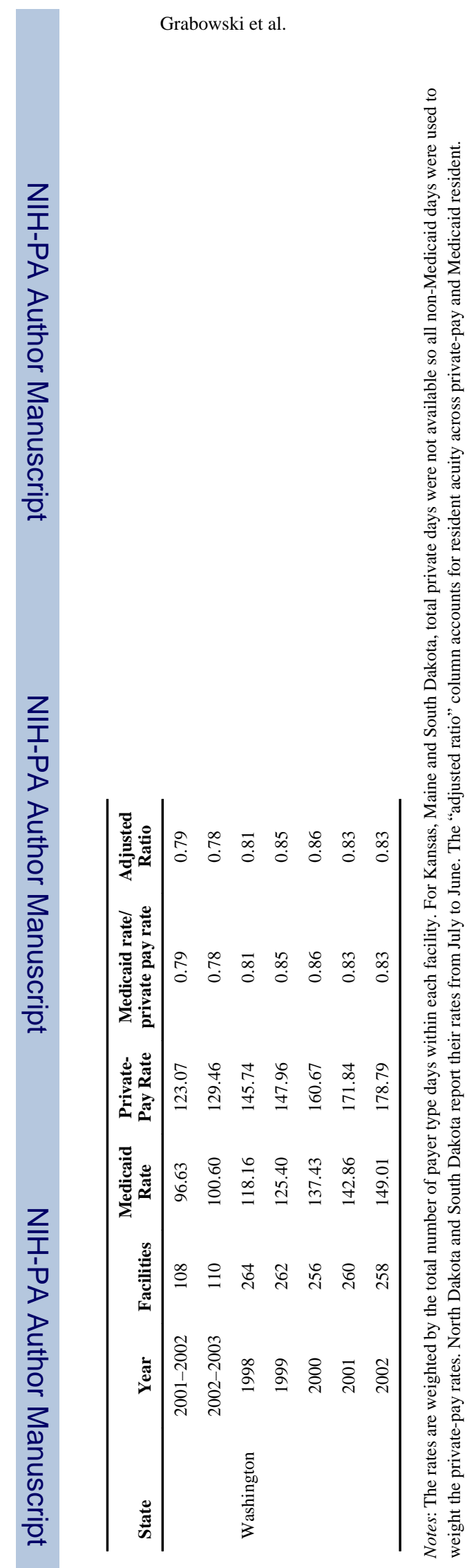

Rev Econ Stat. Author manuscript; available in PMC 2010 May 11. 
Table 3

Medicaid and Nursing Home Quality

\begin{tabular}{|c|c|c|c|}
\hline Outcomes & $\begin{array}{l}\text { No Fixed Effects } \\
\text { (1) }\end{array}$ & $\begin{array}{l}\text { With Nursing Home } \\
\text { Fixed Effects } \\
\text { (2) }\end{array}$ & $\begin{array}{l}\text { With Person Fixed } \\
\text { Effects } \\
\text { (3) }\end{array}$ \\
\hline Physical Restraints & 0.0001 & -0.003 & 0.006 \\
\hline \multirow[t]{2}{*}{$(\mathrm{N}=1,564,820)$} & $(0.14)$ & $(2.60)$ & $(3.85)$ \\
\hline & {$[0.001]$} & {$[-0.033]$} & {$[0.067]$} \\
\hline Catheters & -0.009 & -0.008 & -0.012 \\
\hline \multirow[t]{2}{*}{$(\mathrm{N}=1,564,836)$} & (10.85) & $(8.83)$ & (11.81) \\
\hline & {$[-0.113]$} & {$[-0.100]$} & {$[-0.150]$} \\
\hline Bedfast & -0.001 & -0.001 & -0.005 \\
\hline \multirow[t]{2}{*}{$(\mathrm{N}=1,564,841)$} & (2.17) & (1.46) & $(5.33)$ \\
\hline & {$[-0.017]$} & {$[-0.017]$} & {$[-0.083]$} \\
\hline Feeding Tubes & 0.0001 & -0.002 & -0.007 \\
\hline \multirow[t]{2}{*}{$(\mathrm{N}=1,564,833)$} & $(0.13)$ & $(1.80)$ & $(9.18)$ \\
\hline & {$[0.001]$} & {$[-0.029]$} & {$[-0.100]$} \\
\hline Anti-psychotics & 0.014 & 0.009 & 0.004 \\
\hline \multirow[t]{2}{*}{$(\mathrm{N}=892,578)$} & (7.13) & $(4.23)$ & $(1.85)$ \\
\hline & {$[0.074]$} & {$[0.047]$} & {$[0.021]$} \\
\hline Pressure Ulcers & -0.006 & -0.005 & -0.010 \\
\hline \multirow[t]{2}{*}{$(\mathrm{N}=1,380,289)$} & (7.94) & $(6.89)$ & $(6.80)$ \\
\hline & {$[-0.075]$} & {$[-0.063]$} & {$[-0.125]$} \\
\hline Pain & 0.008 & 0.007 & -0.009 \\
\hline \multirow[t]{2}{*}{$(\mathrm{N}=1,380,127)$} & $(6.86)$ & $(5.91)$ & $(4.92)$ \\
\hline & {$[0.062]$} & {$[0.054]$} & {$[-0.069]$} \\
\hline Incontinence & 0.018 & 0.018 & 0.013 \\
\hline \multirow[t]{2}{*}{$(\mathrm{N}=1,422,656)$} & (12.13) & (12.67) & $(6.33)$ \\
\hline & {$[0.033]$} & {$[0.033]$} & {$[0.024]$} \\
\hline Urinary Infections & -0.006 & -0.005 & -0.012 \\
\hline \multirow[t]{2}{*}{$(\mathrm{N}=1,564,825)$} & (8.07) & $(6.70)$ & $(8.33)$ \\
\hline & {$[-0.067]$} & {$[-0.056]$} & {$[-0.133$} \\
\hline Wound Infections & -0.004 & -0.003 & -0.004 \\
\hline \multirow[t]{2}{*}{$(\mathrm{N}=1,564,825)$} & (12.27) & (10.18) & $(6.27)$ \\
\hline & {$[-0.200]$} & {$[-0.150]$} & {$[-0.200]$} \\
\hline Falls & -0.022 & -0.021 & -0.019 \\
\hline \multirow[t]{2}{*}{$(\mathrm{N}=1,564,786)$} & $(24.40)$ & (21.96) & $(9.95)$ \\
\hline & {$[-0.138]$} & {$[-0.131]$} & {$[-0.119]$} \\
\hline Depression & 0.039 & 0.032 & 0.022 \\
\hline \multirow[t]{2}{*}{$(\mathrm{N}=1,563,566)$} & (17.98) & (14.64) & (11.23) \\
\hline & [0.091] & {$[0.074]$} & {$[0.051]$} \\
\hline
\end{tabular}

Notes: Each cell reports the estimated Medicaid coefficients with the absolute values of the t-statistics presented in parentheses. The effect as a percentage of the dependent variable mean is presented in brackets. All models include the covariates listed in Table 1 along with time fixed effects and were estimated using a linear probability model with robust standard errors clustered at the individual level. 
Table 4

Selection on Observables

\begin{tabular}{lccc}
\hline Outcome & $\begin{array}{c}\text { No Fixed Effects } \\
(\mathbf{1})\end{array}$ & $\begin{array}{c}\text { With Nursing Home } \\
\text { Fixed Effects } \\
\mathbf{( 2 )}\end{array}$ & $\begin{array}{c}\text { With Person Fixed } \\
\text { Effects } \\
(\mathbf{3})\end{array}$ \\
\hline Activities of Daily & -0.384 & -0.445 & 0.065 \\
Living Index & $(13.51)$ & $(15.6)$ & $(2.85)$ \\
$(\mathrm{N}=1,565,017)$ & & & \\
Cognitive & 0.063 & -0.008 & 0.049 \\
Performance Scale & $(8.27)$ & $(1.09)$ & $(8.96)$ \\
$(\mathrm{N}=1,622,351)$ & & & \\
\hline
\end{tabular}

Notes: Each cell reports the estimated Medicaid coefficient with the absolute values of the t-statistics presented in parentheses. The effect as a percentage of the dependent variable mean is presented in brackets. All models include the covariates listed in Table 1 along with time fixed effects and were estimated using a linear probability model with robust standard errors clustered at the individual level. 
Table 5

Effect of rate differential on nursing home quality

\begin{tabular}{|c|c|c|c|}
\hline Outcomes & Medicaid & Rate Differential & $\begin{array}{c}\text { Medicaid } \times \text { Rate } \\
\text { Differential }\end{array}$ \\
\hline Physical Restraints & 0.044 & 0.147 & -0.044 \\
\hline$(\mathrm{N}=1,545,271)$ & (2.77) & $(6.60)$ & (2.47) \\
\hline Catheters & -0.059 & -0.013 & 0.053 \\
\hline$(\mathrm{N}=1,545,285)$ & $(5.48)$ & $(0.90)$ & $(4.40)$ \\
\hline Bedfast & -0.008 & 0.037 & 0.004 \\
\hline$(\mathrm{N}=1,545,290)$ & $(0.89)$ & (2.87) & $(0.39)$ \\
\hline Feeding tubes & -0.075 & 0.070 & 0.076 \\
\hline$(\mathrm{N}=1,545,284)$ & (9.09) & $(6.21)$ & $(8.32)$ \\
\hline Anti-psychotics & 0.018 & 0.068 & -0.016 \\
\hline$(\mathrm{N}=886,339)$ & $(0.64)$ & $(1.60)$ & $(0.49)$ \\
\hline Pressure ulcers & -0.035 & -0.018 & 0.029 \\
\hline$(\mathrm{N}=1,366,262)$ & $(2.40)$ & $(0.93)$ & $(1.76)$ \\
\hline Pain & -0.032 & 0.090 & 0.026 \\
\hline$(\mathrm{N}=1,366,104)$ & $(1.73)$ & $(3.63)$ & $(1.28)$ \\
\hline Incontinence & 0.096 & 0.109 & -0.095 \\
\hline$(\mathrm{N}=1,405,839)$ & $(4.45)$ & $(3.70)$ & $(3.91)$ \\
\hline Urinary infections & -0.053 & 0.008 & 0.046 \\
\hline$(\mathrm{N}=1,545,278)$ & $(3.51)$ & $(0.42)$ & $(2.71)$ \\
\hline Wound infections & -0.019 & -0.011 & 0.017 \\
\hline$(\mathrm{N}=1,545,278)$ & $(3.05)$ & $(1.32)$ & $(2.44)$ \\
\hline Falls & -0.176 & -0.052 & 0.178 \\
\hline$(\mathrm{N}=1,545,236)$ & $(8.78)$ & (1.96) & (7.89) \\
\hline Depression & 0.082 & 0.150 & -0.068 \\
\hline$(\mathrm{N}=1,544,080)$ & (3.73) & $(4.92)$ & $(2.75)$ \\
\hline
\end{tabular}

Notes: Each row reports the estimated Medicaid, rate differential (Medicaid rate/private-pay price) and Medicaid*rate differential coefficients with the absolute values of the t-statistics presented in parentheses. All models include the covariates listed in Table 1 along with time fixed effects and were estimated using a linear probability model with robust standard errors clustered at the individual level. 
Table 6

Payer Source at Time of Admission and Quality

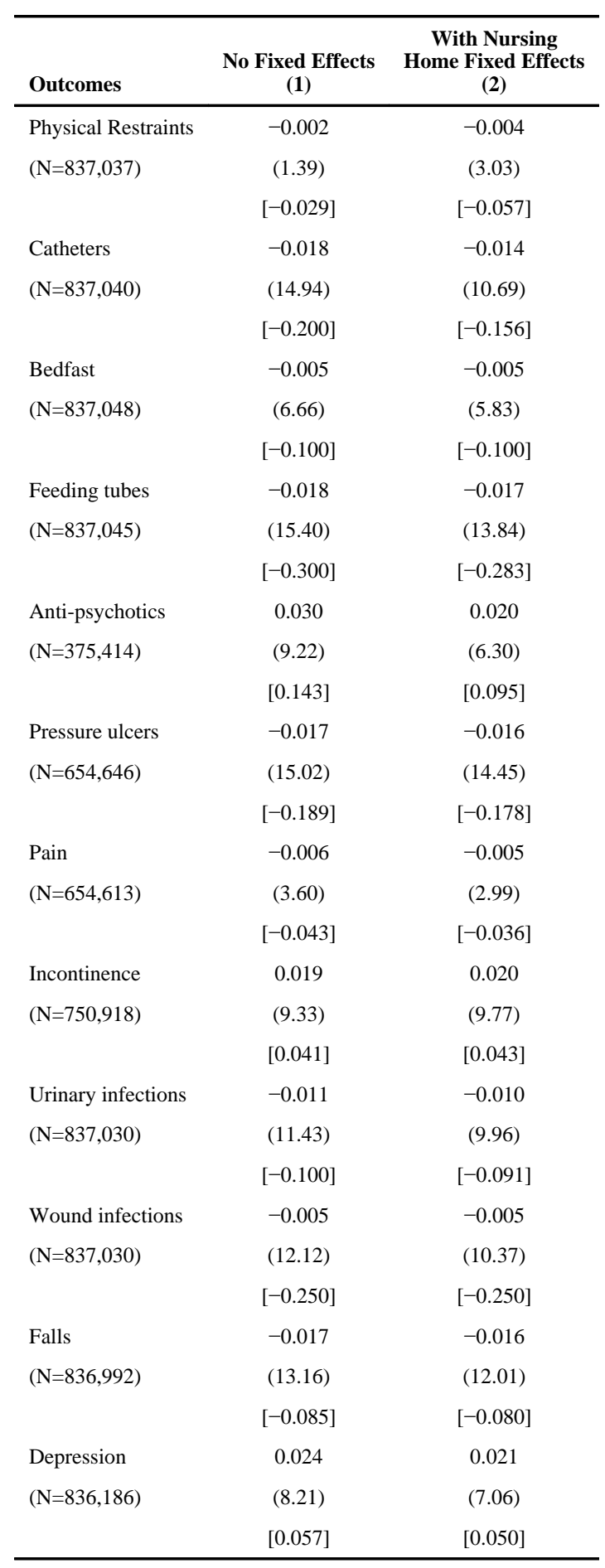

Notes: Each cell reports the estimated Medicaid (at time of admission) coefficient with the absolute values of the t-statistics presented in parentheses. All models include the covariates listed in Table 1 along with time fixed effects and were estimated using a linear probability model with robust standard errors clustered at the individual level. 